\title{
PERSPECTIVES
}

OPINION

\section{Zika virus - reigniting the TORCH}

\section{Carolyn B. Coyne and Helen M. Lazear}

\begin{abstract}
The recent association between Zika virus (ZIKV) infection during pregnancy and fetal microcephaly has led to a renewed interest in the mechanisms by which vertically transmitted microorganisms reach the fetus and cause congenital disease. In this Opinion article, we provide an overview of the structure and cellular composition of the human placenta and of the mechanisms by which traditional 'TORCH' pathogens (Toxoplasma gondii, other, rubella virus, cytomegalovirus and herpes simplex virus) access the fetal compartment. Based on our current understanding of ZIKV pathogenesis and the developmental defects that are caused by fetal ZIKV infection, ZIKV should be considered a TORCH pathogen and future research and public health measures should be planned and implemented accordingly.
\end{abstract}

Zika virus (ZIKV), a member of the Flaviviridae family of RNA viruses, was first isolated in the Zika forest in Uganda in 1947 (REFS 1,2). Prior to 2007, human ZIKV infections were only reported in Africa and Asia, confirmed cases were rare and only mild symptoms, such as rash, fever, headaches, arthralgia and conjunctivitis, were described ${ }^{3-5}$. When ZIKV was first detected in Brazil in early 2015 it came as a surprise, because this obscure mosquito-borne virus had not previously been reported in the Western Hemisphere $^{6,7}$. However, ZIKV had been spreading throughout Oceania in the prior decade and subsequent genetic analysis showed that ZIKV spread to Brazil from the South Pacific ${ }^{8}$. Initial case reports in Brazil were consistent with the fairly benign disease that was reported in previous outbreaks of ZIKV. This changed in the autumn of 2015 when physicians in the north-eastern states of Brazil reported a surge in cases of microcephaly and other congenital abnormalities that coincided with the spread of ZIKV in this region ${ }^{9}$. Since then, it has become evident that ZIKV infection during pregnancy is associated with fetal microcephaly ${ }^{9-16}$. Microcephaly is a severe disorder of fetal brain development that results in a head size that is smaller than normal and is often accompanied by cognitive and physical delays. ZIKV-associated microcephaly, in particular, involves a 'fetal brain disruption sequence' (REFS 13,17,18), which includes growth arrest of the cerebrum, partial collapse of the skull, and distinct folds from redundant scalp skin. ZIKV infections in pregnant women have also been associated with other fetal disorders such as placental insufficiency and fetal growth restriction, ocular disorders, other central nervous system anomalies and even fetal demise ${ }^{19-21}$. These disorders, which together with microcephaly, have been called 'congenital Zika syndrome' (REFS 13,16,21), are reminiscent of those that result from congenital infections caused by 'TORCH' pathogens (this acronym is widely used in the clinic and stands for Toxoplasma gondii, other, rubella virus, cytomegalovirus (CMV) and herpes simplex virus (HSV)). Remarkably, ZIKV can induce fetal damage well beyond the first trimester, as infections even late during pregnancy can result in fetal disease and/or adverse pregnancy outcomes ${ }^{16,19}$.

The emergence of congenital Zika syndrome has led to a renewed interest in the mechanisms by which vertically transmitted microorganisms reach the fetal compartment. In this Opinion article, we discuss the structure of the human placenta, the mechanisms by which other microorganisms that are associated with congenital disease breach the placental barrier and the current models to study ZIKV pathogenesis both in vitro and in vivo. What we know about ZIKV suggests that it should be classified as a TORCH pathogen, so that the experience with traditional TORCH pathogens can inform future research and public health priorities regarding ZIKV.

\section{The placental barrier}

To reach the human fetus, ZIKV must overcome the barrier presented by the placenta, which develops within days of conception and is indispensable for pregnancy.

In the early stages of pregnancy, the human placenta is responsible for anchoring the blastocyst to the maternal endometrium and for establishing the circulatory system that will feed maternal blood directly into the intervillous space (IVS). The IVS is the fluid-filled cavity that bathes the villous tree surfaces of the human placenta, which are formed by syncytiotrophoblasts (SYNs; BOX 1). Owing to their direct contact with maternal blood, SYNs are crucial for protecting the fetus from pathogens. The SYN layer forms a protective barrier even at the earliest stages of pregnancy, with a complete layer of SYNs surrounding the embryo by the end of implantation ( $\sim 7$ days). Defects, even small ones, at any stage of the formation or development of the placenta can lead to miscarriage. Once the uteroplacental circulatory system is fully established, which occurs near the end of the first trimester ( 12 weeks), the placenta is the sole barrier that prevents microorganisms in the maternal blood from accessing the fetal compartment. Earlier, the IVS does not contain maternal blood and is instead filled with clear fluid that is produced by maternal and/or fetal cells ${ }^{22-24}$. Thus, mechanisms of vertical transmission probably differ between the early and later ( $>12$ weeks) stages of human pregnancy.

Vertical transmission of pathogens from mother to fetus can occur by several routes, including: infection of endothelial cells in the maternal microvasculature and spread to invasive extravillous trophoblasts (EVTs), 


\section{Box 1 Structure and function of the human placenta}

In humans, the placenta is haemochorial, meaning that there is direct contact between maternal blood and the placental chorionic villi, which are of fetal origin. Among eutherian mammals, there are two other types of placenta: endotheliochorial (or endochorial), present in carnivores such as cats and dogs, in which the placental villi invade the endothelium of the maternal blood vessels, and epitheliochorial (or epichorial), present in horses, swine, ruminants and whales, in which the chorionic villi contact, but do not invade the maternal endometrium.

The human placenta is composed primarily of specialized cells known as trophoblasts ${ }^{96}$ (see the figure, part a). Covering the villous tree surfaces, and in direct contact with the maternal blood, are syncytiotrophoblasts (SYNs), which are multinucleated, terminally differentiated cells. SYNs are highly polarized and are covered by dense branched microvilli at their apical surfaces, which facilitate the essential exchange of gas, nutrients, and waste that occurs between the mother and fetus throughout pregnancy. Progenitor cytotrophoblasts (CYTs), which are mononuclear and proliferative, underlie the SYN layer and fuse to form the outer syncytium throughout pregnancy. In the later stages of pregnancy, the CYT layer becomes discontinuous given the high demand to replenish the enlarged SYN layer. Similar to humans, the mouse placenta directly contacts maternal blood, but is referred to as haemotrichorial because it contains three trophoblast layers, two of which are SYNs, in contrast to the haemomonochorial human placenta, which has a single SYN layer (see the figure, part b). Whereas other rodent placentas are haemotrichorial, the guinea pig placenta is haemomonochorial, which contributes to the use of guinea pigs as a model for congenital cytomegalovirus (CMV) infection ${ }^{97,98}$.
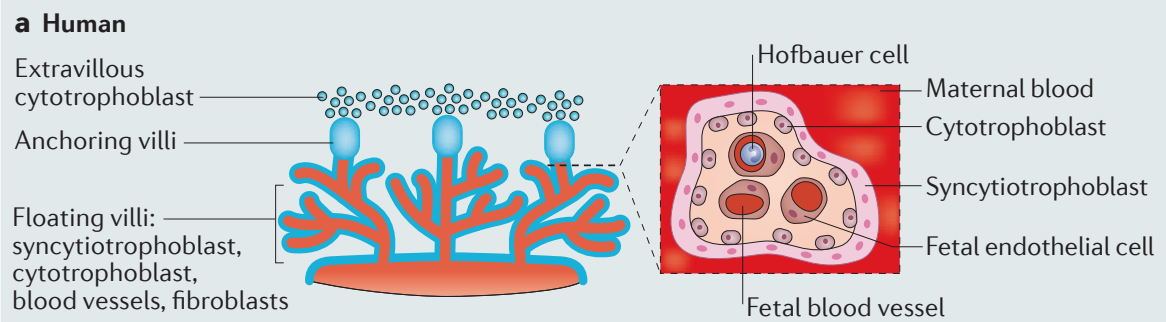

\section{b Mouse}

Fetal blood vessel
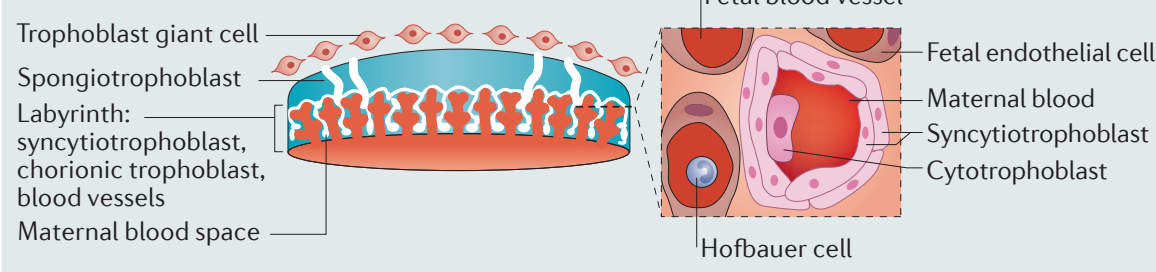

Figure modified from REF. 96, Nature Publishing Group. as a TORCH pathogen, placental infections that are caused by Plasmodium spp., the parasites that are responsible for malaria, are often associated with severe harm to the developing fetus (most commonly growth restriction and/or preterm birth) ${ }^{27}$. Malaria continues to be a source of devastating illness in pregnant women and is responsible for over 200,000 neonatal deaths each year ${ }^{27}$.

The similarities between the congenital disorders that are induced by TORCH pathogens and ZIKV are remarkable, particularly with respect to their neurotropism. Similar to ZIKV, fetal infection by $T$. gondii, rubella virus, $\mathrm{CMV}, \mathrm{VZV}$, and other pathogens, are associated with microcephaly, although the fetal brain disruption sequence seems to be characteristic of ZIKV-associated microcephaly ${ }^{13,16}$. Fetal ZIKV infection produces a cytopathic effect and a lack of inflammation in brain histopathology that are reminiscent of congenital rubella syndrome ${ }^{28}$. The specific congenital malformations that are induced by TORCH pathogens, and probably ZIKV, depend on the gestational age at fetal infection. For example, in the case of rubella virus, infection in the first trimester ( $<12$ weeks) is associated with high rates of miscarriage and with the most severe congenital malformations. As pregnancy progresses, the risk of congenital malformations that result from rubella virus infection decreases and becomes very low during and after the second trimester. Similarly, although the risk of vertical transmission of CMV is highest in the third trimester, the risk of fetal malformations caused by CMV infections is greatest in the first trimester, probably resulting from the key fetal developmental processes that occur at this stage of gestation.

It seems likely that the first trimester ${ }^{15}$ will represent the gestational period when the fetus is most sensitive to ZIKVassociated microcephaly, and a recent study found no adverse outcomes among women who were infected with ZIKV during their third trimester ${ }^{29}$. However, other reports found various adverse outcomes including placental insufficiency, intrauterine growth restriction, cerebral calcifications as well as microcephaly following ZIKV infections at various stages of gestation ${ }^{16,19}$. In addition, it is important to note that ZIKV-induced congenital abnormalities, such as neurological and ocular disorders, can present without microcephaly ${ }^{16,30}$. Most alarmingly, fetal demise has been reported in women who were infected as late as 


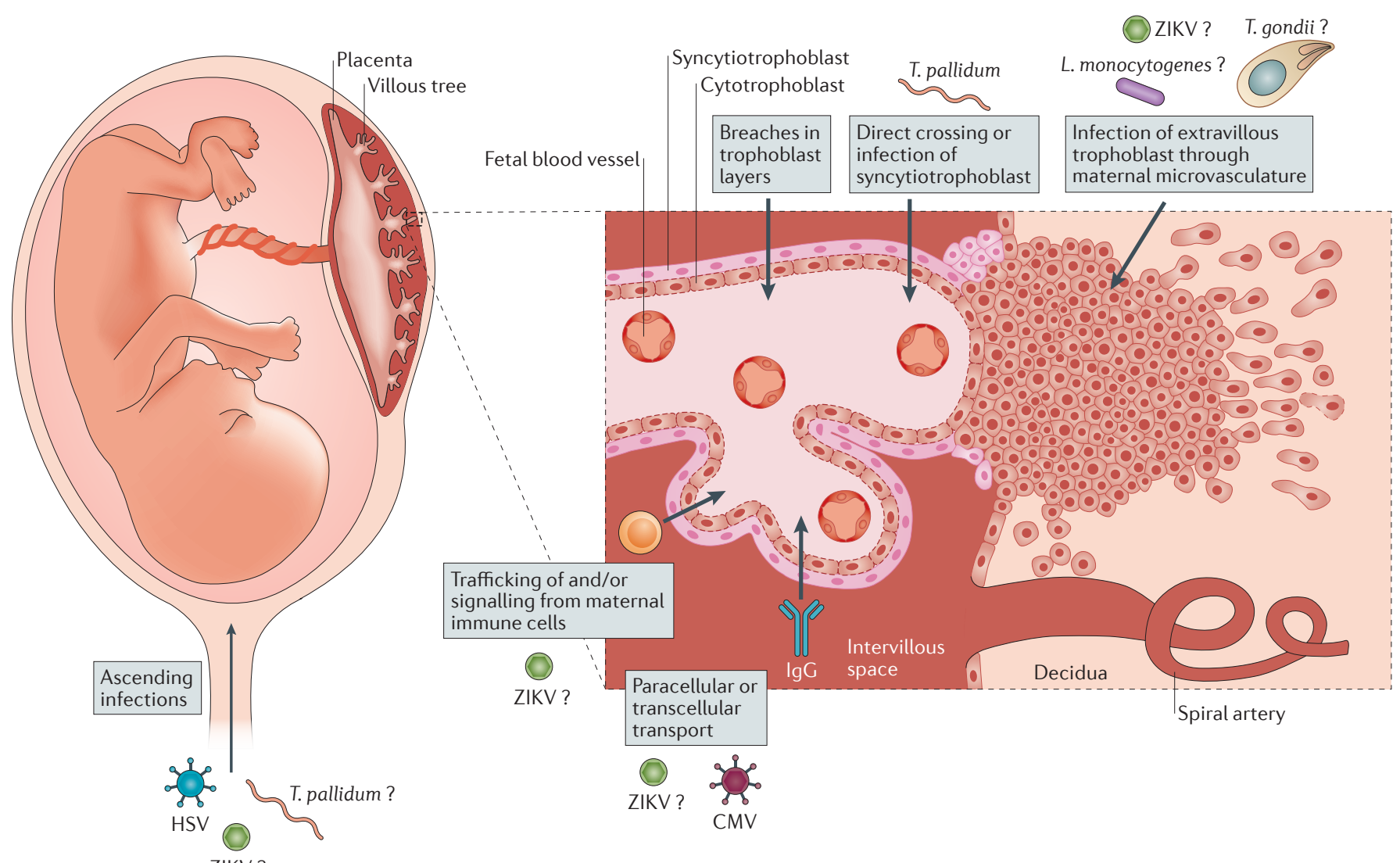

Figure 1 | Routes used by TORCH pathogens to overcome the placental barrier. There are several pathways by which Zika virus (ZIKV), and other TORCH (Toxoplasma gondii, other, rubella virus, cytomegalovirus (CMV), herpes simplex virus (HSV)) pathogens, might be vertically transmitted. Pathogens might invade by several routes and these routes can vary depending on the gestational stage. The precise routes that are used by different pathogens remain largely unknown. Ascending infection with HSV and possibly by Treponema pallidum might expose the placenta to these pathogens. In the case of both T. gondii and Listeria monocytogenes, studies using human first trimester explants suggest that extravillous trophoblasts (EVTs) are the 'Achilles heel' that enables the delivery of these pathogens to the fetal compartment, without infection of syncytiotrophoblasts (SYNs). The mechanisms by which rubella virus and $\mathrm{CMV}$ cross the placental barrier are less clear, although CMV might be transported across the trophoblast layers by antibody-dependent transcytosis without replication in the trophoblast layers. It is unknown how ZIKV crosses the placental barrier. Potential routes of vertical transmission include: breaks in the SYN layer (which could result from maternal immune signals), exposing the more susceptible cells of the villous core; direct infection of the SYN layer; bypassing the SYN layer through non-replicative routes (such as antibody-mediated transcytosis); infection of EVTs or other more permissive placental cell types, most probably through the maternal microvasculature and/or decidua; delivery to the fetus and/or villous core through maternal cells (most likely those of immune origin); and/or ascending intravaginal infection. IgG, immunoglobulin G.
25 weeks and 32 weeks of gestation, which suggests that ZIKV can have devastating fetal consequences at various gestational ages $^{19}$. Furthermore, ZIKV-associated microcephaly has been reported even in mothers who have asymptomatic ZIKV infection ${ }^{16,29}$, which suggests that the severity of maternal illness may not predict fetal outcomes. Prospective longitudinal studies are required to better define the manifestations of congenital ZIKV syndrome, identify risk factors that contribute to adverse outcomes and to determine the effect of ZIKV infection at different stages of gestation. Such efforts are already underway: the United States National Institutes of Health and Brazilian Fundacao Oswaldo Cruz-Fiocruz recently initiated a large study focused on Puerto Rico, Brazil and Colombia, and other studies are planned and in progress ${ }^{31}$.

\section{Mechanisms of vertical transmission}

The mechanisms of vertical transmission of TORCH pathogens remain largely unknown. For both T. gondii and L. monocytogenes, studies in first trimester human explant models indicate that the SYN layer forms an effective barrier to the passage of both pathogens ${ }^{32,33}$. Given the broad antiviral resistance of primary SYNs isolated from full-term placentas ${ }^{34-36}$, it seems likely that viruses access the fetus through routes that do not involve replication in the SYN layer, at least at later stages of pregnancy. EVTs may be an entry portal for microorganisms that enables them to bypass the SYN barrier ${ }^{32,33,37}$, as suggested in a recent study of ZIKV that used first trimester explants and second trimester primary cells ${ }^{38}$. However, it remains unclear how pathogens reach these cells, as EVTs are buried in the decidua; possibly these cells are exposed to maternal blood during vascularization and/or contact infected maternal immune cells. Although SYNs that are isolated from full-term placentas and SYNs that are exposed to virus from first trimester explants resist ZIKV infection ${ }^{36,38}$, it is possible that ZIKV replicates in SYNs at very early stages of gestation, or that the virus targets other placental cells, such as EVTs. ZIKV RNA and antigens have been detected in the placentas of fetuses with 
Table 1 | Models to study Zika virus (ZIKV) teratogenicity

\begin{tabular}{|c|c|c|c|}
\hline Description & Use as model of ZIKV & Advantages and disadvantages & References \\
\hline \multicolumn{4}{|l|}{ In vitro } \\
\hline $\begin{array}{l}\text { Primary human trophoblasts } \\
\text { from full-term placentas }\end{array}$ & $\begin{array}{l}\text { Study possible mechanisms } \\
\text { of vertical transmission; } \\
\text { cell biology of, and innate } \\
\text { immunity to, infection }\end{array}$ & $\begin{array}{l}\text { - Advantages: Primary human cells, including SYNs and CYTs; } \\
\text { recapitulate many of the unique morphological and functional } \\
\text { features of trophoblasts in vivo; easy to obtain (after delivery) } \\
\text { - Disadvantages: Accurately reflect only the second half of pregnancy; } \\
\text { not tractable; short life span in culture }\end{array}$ & 36 \\
\hline Placental villous tree explants & $\begin{array}{l}\text { Study possible mechanisms } \\
\text { of vertical transmission; } \\
\text { cell biology of, and innate } \\
\text { immunity to, infection }\end{array}$ & $\begin{array}{l}\text { - Advantages: Accurately models the morphology and cellular } \\
\text { composition of the human placenta; can be used to assess the } \\
\text { susceptibility of specific placental cell types that are targeted by ZIKV; } \\
\text { can study early gestation placentas } \\
\text { - Disadvantages: Can be difficult to obtain (particularly from first } \\
\text { and second trimesters); not tractable; short life span in culture }\end{array}$ & 38 \\
\hline $\begin{array}{l}\text { Human placental cell lines } \\
\text { (for example, BeWo, JEG-3, } \\
\text { JAR and HTR8) }\end{array}$ & $\begin{array}{l}\text { Assess viral replication } \\
\text { and test therapeutics }\end{array}$ & $\begin{array}{l}\text { - Advantages: Permissive to ZIKV infection; ease of use; some can be } \\
\text { induced to form syncytia (BeWo); tractable } \\
\text { - Disadvantages: Do not reflect the antiviral properties of primary } \\
\text { placental cells; do not spontaneously fuse to form SYNs (exception is } \\
\text { JEG-3 cells in 3D culture) }\end{array}$ & $36,40,105$ \\
\hline Human neural stem cells & $\begin{array}{l}\text { Study viral infection } \\
\text { and neurotropism; } \\
\text { test therapeutics }\end{array}$ & $\begin{array}{l}\text { - Advantages: Reliable model for studying neurotropism; embryonic } \\
\text { and fetal cells can be expanded easily } \\
\text { - Disadvantages: Access to cells may be limited; not tractable }\end{array}$ & 62,64 \\
\hline \multicolumn{4}{|l|}{ In vivo } \\
\hline 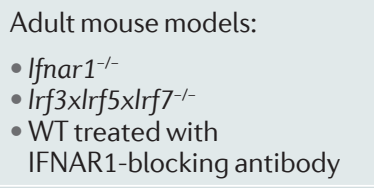 & $\begin{array}{l}\text { Model pathogenesis; test } \\
\text { vaccines and therapeutics }\end{array}$ & $\begin{array}{l}\text { - Advantages: Recapitulate several aspects of human disease; } \\
\text { animal models can be used to study various aspects of pathogenesis } \\
\text { and vaccine responses } \\
\text { - Disadvantages: Pathogenesis requires ablation of IFNa and IFN } \beta \\
\text { signalling, limiting studies of antiviral immunity }\end{array}$ & $68-71$ \\
\hline Mouse intrauterine infection & $\begin{array}{l}\text { Study fetal pathogenesis } \\
\text { directly }\end{array}$ & $\begin{array}{l}\text {-Advantages: Directly study teratogenic effects independent of } \\
\text { maternal infection and transplacental transmission; better control } \\
\text { of fetal infection } \\
\text { - Disadvantages: Does not model transplacental transmission }\end{array}$ & 72,75 \\
\hline $\begin{array}{l}\text { Non-human primates: } \\
\text { - Rhesus macaque } \\
\text { - Cynomologous macaque }\end{array}$ & $\begin{array}{l}\text { Understand pathogenesis } \\
\text { and vertical transmission; } \\
\text { test vaccines and } \\
\text { therapeutics }\end{array}$ & $\begin{array}{l}\text { - Advantages: Placenta more closely resembles human placenta; } \\
\text { immune competent animals develop disease } \\
\text { - Disadvantages: Cost, small sample size; not tractable }\end{array}$ & 59 \\
\hline
\end{tabular}

CYT, cytotrophoblast; IFN $\alpha$, interferon- $\alpha$; IFNAR, interferon $\alpha / \beta$ receptor; IRF, interferon regulatory factor; SYN, syncytiotrophoblast; WT, wild-type; ZIKV, Zika virus.

microcephaly ${ }^{28,39}$, and a mouse model of ZIKV vertical transmission found high viral loads in the placenta, as well as viral genomes and viral particles in placental trophoblasts $^{40}$ (TABLE 1). These findings, coupled with more recent work that used first trimester human placental explants $\mathrm{s}^{38}$, are consistent with a model in which ZIKV from the maternal circulation infects EVTs and then spreads to the fetus. However, the anatomical and immunological differences between the mouse and human placentas may limit the direct applicability of results from mouse studies to humans ${ }^{41,42}$ (BOX 1). In the same mouse model, ZIKV triggered apoptosis and vascular damage in the placenta, which could contribute to vertical transmission by directly damaging the trophoblast layers. Fetal-derived macrophages (Hofbauer cells) are susceptible to ZIKV infection and might be important for vertical transmission ${ }^{38,43}$. Furthermore, ZIKV antigens were detected in Hofbauer cells from fetuses with microcephaly ${ }^{28}$.
However, given that Hofbauer cells are not in direct contact with the maternal circulation and are primarily located close to fetal capillaries, pathogens still require a mechanism to cross the SYN layer of the placenta before accessing these cells. A recent comprehensive study that used first trimester human placental explants found that several placental cell types might be targeted by ZIKV early in gestation, including both cytotrophoblasts (CYTs) and EVTs, which suggests that there may be several routes for 
ZIKV vertical transmission ${ }^{38}$. These could involve viral replication in select placental cell populations, and/or non-replicative routes such as in trafficking immune cells or by transcytosis. Furthermore, the maternal immune response might damage the placenta, which may allow viral passage. Infected maternal cells could deliver ZIKV to the decidua, which may lead to the infection of EVTs. Transmigration of infected maternal immune cells could also deliver ZIKV to the fetus, similarly to the transcytosis of fetal cells into the maternal circulation ${ }^{44}$. The transfer of maternal immunoglobulin $\mathrm{G}$ ( $\operatorname{IgG}$ ) is crucial to protect the developing fetus, particularly during the stages when the fetus is most sensitive to viral infections. However, in some cases, this process may expose the fetus to pathogens; for example, maternal IgG antibodies might carry CMV across the SYN layer through the neonatal Fc receptor for $\operatorname{IgG}(\mathrm{FcRn})^{45}$. Among flaviviruses, the ability of antibodies to facilitate viral infection of FcR-bearing cells is well established for DENV, a process termed antibody-dependent enhancement ${ }^{46,47}$. There are four serotypes of DENV, and secondary infection with a heterologous serotype can result in exacerbated disease, as cross-reactive non-neutralizing antibodies promote FcR-mediated infection of myeloid cells. Furthermore, severe disease is also seen during primary DENV infections in infants, as infection is enhanced by circulating maternal antibodies ${ }^{48}$. Given the emergence of ZIKV in areas with high DENV seroprevalence, and the substantial antigenic cross-reactivity between ZIKV and $\mathrm{DENV}^{49-53}$, it is plausible that maternal DENV antibodies could contribute to ZIKV placental invasion through FcRn-mediated transcytosis. However, it is worth noting that transplacental delivery of maternal IgG is quite low during the first trimester (before the establishment of a haemochorial placenta), with levels increasing in the second and third trimesters ${ }^{54-58}$. In vitro studies have demonstrated that DENV immune sera and DENV monoclonal antibodies can enhance ZIKV infection in cell culture, but the importance of this in vivo, at physiological antibody concentrations, and in placental cells specifically, remains to be determined ${ }^{50,51}$. Finally, ZIKV RNA has been detected in the female genital tract in humans and in rhesus macaques ${ }^{59,60}$, which raises the possibility that ZIKV could access the fetal compartment by transvaginal ascending infection, in addition to transplacental haematogenous spread.

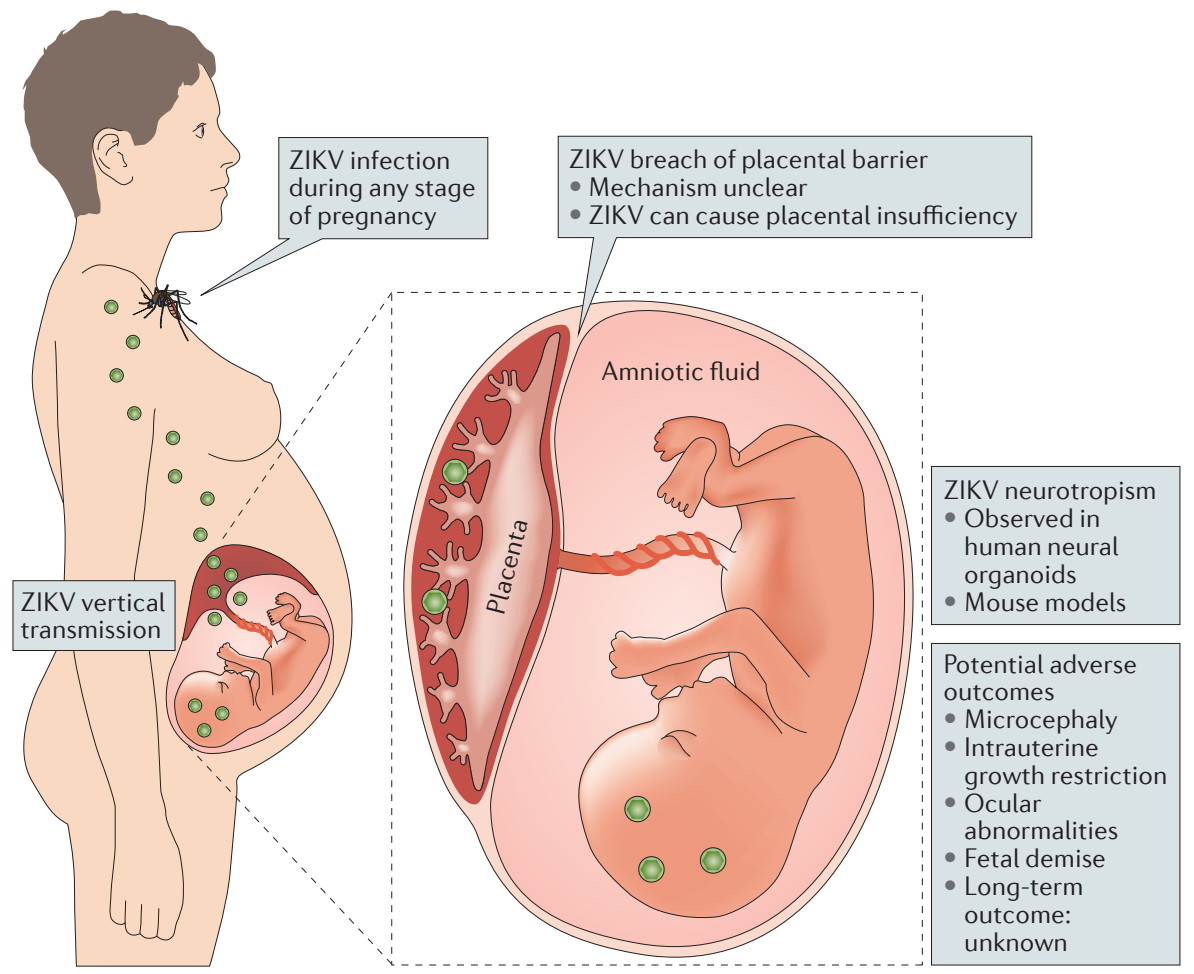

Figure 2 | Vertical transmission and congenital disease induced by ZIKV. After crossing the placental barrier, Zika virus (ZIKV) infection can have devastating consequences for the developing fetus. Of note, unlike many TORCH (Toxoplasma gondii, other, rubella virus, cytomegalovirus (CMV) and herpes simplex virus (HSV)) pathogens, some evidence suggests that ZIKV can induce fetal disease and/or other adverse pregnancy outcomes throughout pregnancy. Potential adverse outcomes of fetal ZIKV infection include microcephaly, hydranencephaly, growth restriction, ocular abnormalities and death, whereas the virus causes mild or no symptoms in the pregnant mother. In addition, it is important to note that adverse outcomes due to in utero infections with some TORCH pathogens do not present until months or years after birth. Human organoid and mouse models support the destructive nature of ZIKV infections in the fetal brain.

\section{Zika virus pathogenesis}

As almost no laboratory research was carried out on ZIKV before the current epidemic, the pathogenic mechanisms of ZIKV are only beginning to be understood (FIG. 2). Aspects of ZIKV disease in humans, such as teratogenicity and sexual transmission, are unexpected based on extensive experience with related flaviviruses, which highlights the need to better understand this emerging pathogen ${ }^{2}$.

The mechanisms that enable ZIKV to cross the placenta and cause fetal damage are of particular interest and importance. It remains unclear how ZIKV gains access to the fetal compartment, although possible mechanisms are discussed above. The full range of fetal tissues that are targeted by ZIKV remains to be determined, but ZIKV antigens and genomes were detected in the brains of infants and fetuses with microcephaly, without being detectable in other organs ${ }^{28,39,61}$. ZIKV infects neuroprogenitor cells in culture $^{62,63}$, which is consistent with damage to the developing brain, although the outcome may depend on the gestational age when infection occurs. In vitro models using human stem cell-derived neural progenitors (TABLE 1) have confirmed the destructive nature of ZIKV infection in neural cells ${ }^{62,64-67}$. Such neurotropism is more characteristic of encephalitic flaviviruses (for example, West Nile virus and Japanese encephalitis virus) than of viruses such as DENV, to which ZIKV is more closely related. Although ZIKV typically causes an acute infection with viraemia cleared within one week, there is evidence of persistent maternal viraemia in the presence of a fetus infected with ZIKV ${ }^{39}$ and similar persistence was observed in pregnant rhesus macaques ${ }^{59}$. It is unclear whether this results from persistent viral shedding from the fetus or is a consequence of changes in the maternal immune system during pregnancy.

New small-animal models have been developed to study ZIKV infection in vivo (TABLE 1). These models recapitulate many 
aspects of ZIKV-induced disease in humans, such as infection of the brain, testes and fetus ${ }^{40,67-71}$. Recent reports demonstrate transplacental transmission of ZIKV in pregnant mice and associated fetal abnormalities including microcephaly, placental insufficiency, intrauterine growth restriction and fetal demise, all of which are relevant to congenital ZIKV infection in humans ${ }^{40,67,72}$. Mouse models of ZIKV pathogenesis and vertical transmission are crucial for understanding ZIKV pathogenesis and for testing candidate vaccines and antivirals. However, differences in anatomy and immune mechanisms between mouse and human placentas may limit the direct applicability of findings in mouse models to human disease $\mathrm{e}^{73}$ (BOX 1). For example, human SYNs that are isolated from full-term placentas constitutively produce interferon- $\lambda 1$ (IFN $\lambda 1$; also known as type III interferon or interleukin-29 (IL-29)), which protects cells from ZIKV infection in both a paracrine and autocrine manner ${ }^{36}$. However, mice do not express IFN $\lambda 1$ (REF. 74); thus, the role of IFN $\lambda$ in the protection of the murine placenta may be distinct from that in humans. Moreover, most existing mouse models of ZIKV pathogenesis use mice that lack the type I interferon receptor (for example, Ifnar $1^{-1-}$ mice) and thus have severely impaired antiviral responses ${ }^{68-71}$. Mice that have intact IFNa and IFN $\beta$ signalling show minimal viraemia and no disease signs or lethality after ZIKV infection. By contrast, transplacental transmission of ZIKV was demonstrated in Ifnar ${ }^{-1-}$ dams that were mated with wild-type sires, such that the affected fetuses and placentas would have intact IFNa and IFN $\beta$ signalling (Ifnar 1 $\left.^{+-}\right)^{40}$. Vertical transmission has also been demonstrated in wild-type SJL and C57Bl/6 mice (67,72 $^{\text {, although }}$ this required higher infection doses; ablated maternal IFN $\alpha$ and IFN $\beta$ signalling and high infection doses probably increase maternal viraemia to the level that is required for transplacental transmission.

Other groups have used direct intrauterine injection of mice to study the fetal pathogenesis of ZIKV ${ }^{72,75}$; however, this system does not model transplacental transmission from a viraemic mother. ZIKV infection in non-human primates, including rhesus macaques, mimics many aspects of human disease and, importantly, immune competent animals develop signs of disease $\mathrm{e}^{1,59,76}$. Furthermore, the macaque placenta is similar to the human placenta and so serves as a better model of congenital infection than mice. Ongoing studies of
ZIKV infection in pregnant rhesus macaques are likely to provide additional insight into the mechanisms of ZIKV transplacental transmission and teratogenicity ${ }^{59}$. In one study, pregnant mice that were infected with DENV did not show transplacental transmission and fetal damage as seen with ZIKV infection ${ }^{40}$. However, most animal and cell culture studies of ZIKV transplacental transmission and fetal neuropathogenesis have not directly compared ZIKV infection with related viruses, such as DENV, that are not generally associated with congenital infections in humans. Furthermore, congenital infection has only been apparent during the current ZIKV epidemic in the Americas; it is not known whether historical isolates of $\mathrm{ZIKV}$, or contemporary isolates from different sources, share this property. Thus, the properties that determine ZIKV teratogenicity in humans, and how faithfully pathogenesis is recapitulated in current models, remain unclear.

\section{Is ZIKV a TORCH pathogen?}

The association between ZIKV and fetal microcephaly was unexpected, as flaviviruses are not generally associated with congenital infection. For comparison, maternal DENV infection during pregnancy is associated with adverse outcomes, including preterm birth and low birth weight ${ }^{77}$, which may be manifestations of maternal disease. However, there is no evidence of birth defects caused by DENV, despite millions of infections worldwide each year ${ }^{78,79}$. Although many unanswered questions remain, a growing body of clinical, epidemiological and molecular evidence supports a causal role for congenital ZIKV infection in the development of microcephaly ${ }^{13}$. The present evidence includes a spatiotemporal association between cases of microcephaly and ZIKV infections ${ }^{9}$; case-control studies of ZIKV-infected pregnant women in Brazil $^{19}$; the detection of infectious ZIKV and complete ZIKV genomes in tissues of affected infants and fetuses ${ }^{14,39,61}$; cell culture and animal model studies that demonstrate ZIKV infection in relevant cells and tissues ${ }^{40,59,64,67,72}$; and a lack of other plausible explanations for the observed increase in cases of microcephaly in north-eastern Brazil. Although the emergence of ZIKV in Brazil coincided with an increase in cases of microcephaly, the total number of cases and the exact increase over baseline levels remain to be determined ${ }^{16}$. Ongoing research will provide a clearer picture of the rate at which maternal ZIKV infection results in transplacental infection and fetal damage, as well as how that risk changes during the course of gestation. Understanding these risks is crucial for pregnant women to make informed decisions and for the public health response to ZIKV.

The available evidence suggests that ZIKV should be considered a new member of the TORCH family of pathogens and the expanding category of 'other' invites a new acronym to describe teratogenic pathogens. For example, it has been proposed that the term TORCH be amended to 'TORCHES' or 'STORCH' to directly include syphilis, which is caused by T. pallidum ${ }^{80}$. Given the severe congenital anomalies that are associated with ZIKV, it may be appropriate to more directly include ZIKV in the acronym, such as using TORCHZ or another abbreviation. Unlike other TORCH pathogens, which are generally transmitted through person-to-person contact or through contaminated food, ZIKV typically is mosquito-borne and therefore requires substantially different public health control measures than other TORCH pathogens (BOX 2). ZIKV can also be sexually transmitted ${ }^{81-86}$. Some evidence suggests that men shed infectious ZIKV in semen for weeks or months after the acute infection is resolved ${ }^{82,87}$, potentially acting as long-term transmission reservoirs. ZIKV RNA has been detected in the female reproductive $\operatorname{tract}^{37}$, although, to date, there has been a single report of female-to-male sexual transmission ${ }^{86}$ compared with dozens of reported cases from men. The contribution of sexual transmission compared with mosquito-borne transmission to outbreaks of ZIKV is unclear. A higher incidence of ZIKV infection has been reported in women than in men ${ }^{29,88}$, which could be caused by male-to-female sexual transmission augmenting mosquito-borne transmission to both sexes. However, other explanations include enhanced ZIKV awareness and surveillance among women owing to its association with birth defects; a greater willingness of women to seek medical care; an innate susceptibility of women due to immunological or other factors; and/or greater exposure to the anthropophilic domestic mosquitoes that transmit ZIKV. Furthermore, the role of sexual transmission for ZIKV congenital disease is unclear; if the virus reaches the fetal compartment through haematogenous spread, the initial route by which the virus infects the mother may not matter. Many questions remain regarding the teratogenic mechanisms of ZIKV, and experience with other TORCH 


\section{Box 2 | Public health implications}

To date, by far the largest number of confirmed Zika virus (ZIKV)-associated cases of microcephaly (more than 1,600 (REF. 99)) have occurred in Brazil, particularly in the north-eastern region of the country where ZIKV first emerged. More recently, ZIKV-associated cases of microcephaly have been reported in other Latin American countries, including Colombia and Panama. In the United States and Europe, ZIKV-affected pregnancies (including cases of microcephaly) have occurred among women who were infected with ZIKV in Latin America or the Caribbean ${ }^{39,61,10,101}$. Thus far, the number of ZIKV-affected pregnancies outside of Brazil has remained small, which probably reflects the lower total number of infections in other countries and the timing of ZIKV spread throughout the region. As time goes on, the rates and characteristics of ZIKV-affected pregnancies in different regions will increase our understanding of the factors that contribute to ZIKV transplacental transmission and teratogenicity.

As awareness of the threat that is posed by ZIKV infection during pregnancy spreads, and as the evidence of the teratogenic effects of ZIKV increases, rates of ZIKV-affected pregnancies may change depending on the decisions that are made by pregnant women in areas where ZIKV is circulating ${ }^{102}$. The United States Centers for Disease Control and Prevention (CDC), as well as other public health agencies worldwide, have advised pregnant women to avoid travel to areas where ZIKV is circulating and to avoid unprotected sex with male partners who may have been exposed to ZIKV ${ }^{103}$. In ZIKV-endemic areas, pregnant women have been advised to protect themselves from mosquitoes, and several countries have advised women to avoid pregnancy altogether until the ZIKV epidemic subsides. Both recommendations suffer from considerable obstacles to implementation. In particular, access to contraception and prenatal services are important aspects of a public health response to ZIKV. The ZIKV epidemic also has focused attention on access to safe abortion services, which is reminiscent of the epidemic of congenital rubella syndrome in the United States in the 1960s. Although no link to microcephaly was noted during the 2013-2014 outbreak of ZIKV in French Polynesia, a retrospective analysis identified eight cases of ZIKV-associated microcephaly, five of which resulted in elective termination at a median age of 30 weeks gestation; a different retrospective analysis of this outbreak found that 11 out of 14 pregnancies with severe cerebral malformations were terminated ${ }^{10,89}$. Other reports have described ZIKV-affected pregnancies that resulted in elective termination in Slovenia, Spain and the United States ${ }^{39,61,100}$. The legal status of abortion varies widely across Latin America and the Caribbean, including being entirely illegal (El Salvador and Nicaragua), highly restricted (Brazil and Venezuela) and more permissive (Colombia and Puerto Rico) ${ }^{104}$. Varying abortion access (whether de jure or de facto) could affect observed rates of microcephaly in live-born infants across the region.

pathogens should inform studies of the risk factors, transmission mechanisms and pathological effects of ZIKV.

Microcephaly was the first congenital abnormality that was associated with ZIKV infection, probably owing to its dramatic presentation ${ }^{9}$. However, similar to other TORCH pathogens, ZIKV probably causes a range of developmental abnormalities. The characteristics of congenital Zika syndrome are beginning to be described and include microcephaly, lissencephaly, ventriculomegaly, hydranencephaly, ocular abnormalities, placental insufficiency and fetal loss ${ }^{13,16,21,28}$. The long-term manifestations of TORCH infections may not be apparent at birth and often are diagnosed as the child develops. It will take time before the full range of ZIKV-associated birth defects becomes apparent, especially for more subtle effects such as hearing loss, cognitive and behavioural impairments, or other complications.

The available evidence indicates that ZIKV infection during pregnancy can result in transplacental transmission and fetal damage. However, we do not yet know whether ZIKV infection is sufficient for these effects, or whether additional factors contribute. No such cofactors that potentiate ZIKV teratogenicity have been identified, but these could include environmental factors, virus strain differences, co-infections, maternal immune responses and/or host genetics. It is unclear why ZIKV has been associated with birth defects during the current epidemic in Latin America, but not during historical outbreaks. The simplest explanation is that this ZIKV outbreak is vastly larger than previous ones: millions of people are estimated to have been infected in Latin America, compared with $\sim 100,000$ in French Polynesia in 2013-2014, $\sim 5,000$ in Micronesia in 2007 and only a handful of human cases reported in prior decades $^{88,89}$. However, we know very little about the viral, host and environmental factors that contribute to ZIKV pathogenesis and additional studies are required.

\section{Final conclusions}

Many groups are focused on developing vaccines and antivirals to combat ZIKV, but a challenge is that these interventions must target pregnant women and/or couples who are planning to conceive (BOX 2). The difficulty of testing new (and old) medications in pregnant women is a general problem; the reluctance to pursue such studies has resulted in a dearth of information regarding drug safety and efficacy during pregnancy, and is an obstacle to adequate prenatal care. Evaluation in pregnant women will be a crucial component of new ZIKV interventions and the development of innovative approaches to conduct these studies ethically may benefit maternal health more generally. There are currently very few effective treatments to prevent the vertical transmission of viral infections during pregnancy, although some antiviral therapies that reduce viral burden in the mother, such as antiretroviral therapies in HIV-positive women, can reduce viral loads to lessen the risk of vertical transmission ${ }^{90,91}$. Developing new therapeutic strategies to prevent vertical transmission of ZIKV and other TORCH pathogens will require a better understanding of the mechanisms that are used by these microorganisms to cross the placenta and cause fetal disease.

The long-term picture of ZIKV in the Western Hemisphere is unclear. Model-based predictions suggest that more than 1.5 million women may be at risk of contracting ZIKV during pregnancy and that in total $\sim 90$ million individuals are at risk of infection during the current epidemic $^{92}$. The unprecedented size of the current epidemic is probably a consequence of the emergence of the virus in an immunologically naive population, and the incidence of ZIKV infection is likely to decline in the presence of substantial population immunity. There is no evidence that prior ZIKV infection affects future pregnancies, thus the rates of microcephaly and other ZIKV-associated birth defects may decrease if most women are exposed to ZIKV before reaching child-bearing age. However, experience in Latin America with DENV and chikungunya virus, which are spread by the same mosquito vectors as ZIKV, suggests that continued ZIKV outbreaks and adult infections are likely. This highlights the need for a vaccine to ensure that women of child-bearing age are immune to ZIKV, much as routine immunization with the measlesmumps-rubella vaccine has eliminated congenital rubella syndrome from the Americas. However, amid all of the attention that is focused on ZIKV-associated birth defects, it is worth remembering that each 
year there are more than $\sim 100,000$ cases of congenital rubella syndrome, $\sim 200,000$ cases of congenital toxoplasmosis and $\sim 30,000$ cases of congenital CMV worldwide $\mathrm{e}^{93-95}$. The ongoing outbreak of ZIKV highlights the need not only to better understand how ZIKV and other TORCH pathogens breach the placental barrier and induce congenital disease, but also the urgent need to assess the safety and efficacy of antimicrobial therapeutics in pregnant women.

Carolyn B. Coyne is at the Department of Microbiology and Molecular Genetics, University of Pittsburgh, Pittsburgh, Pennsylvania 15219, USA; and the Magee-Womens Research Institute, Department of Obstetrics, Gynecology, and Reproductive Science, University of Pittsburgh, Pittsburgh, Pennsylvania 15213, USA.

Helen M. Lazear is at the Department of Microbiology and Immunology, University of North Carolina at Chapel Hill, Chapel Hill, North Carolina 27599, USA.

Correspondence to C.B.C. and H.M.L. coynec2@pitt.edu; helen.lazear@med.unc.edu

doi: $10.1038 /$ nrmicro.2016.125 Published online 30 Aug 2016

1. Dick, G. W., Kitchen, S. F. \& Haddow, A. J. Zika virus. I. Isolations and serological specificity. Trans. $R$. Soc. Trop. Med. Hyg. 46, 509-520 (1952).

2. Lazear, H. M. ¿ Diamond, M. S. Zika virus: new clinical syndromes and its emergence in the Western hemisphere. J. Virol. 90, 4864-4875 (2016).

3. Fauci, A. S. \& Morens, D. M. Zika virus in the Americas - yet another arbovirus threat. N. Engl. J. Med. 374, 601-604 (2016).

4. Simpson, D. I. Zika virus infection in man. Trans. R. Soc. Trop. Med. Hyg. 58, 335-338 (1964).

5. Bearcroft, W. G. Zika virus infection experimentally induced in a human volunteer. Trans. R. Soc. Trop. Med. Hyg. 50, 442-448 (1956).

6. Zanluca, C. et al. First report of autochthonous transmission of Zika virus in Brazil. Mem. Inst. Oswaldo Cruz 110, 569-572 (2015).

7. Campos, G. S., Bandeira, A. C. \& Sardi, S. I. Zika virus outbreak, Bahia, Brazil. Emerg. Infect. Dis. 21, 1885-1886 (2015).

8. Faria, N. R. et al. Zika virus in the Americas: Early epidemiological and genetic findings. Science 352 , 345-349 (2016)

9. Schuler-Faccini, L. et al. Possible association between Zika virus infection and microcephaly - Brazil, 2015. MMWR. Morb. Mortal. Wkly Rep. 65, 59-62 (2016).

10. Cauchemez, S. et al. Association between Zika virus and microcephaly in French Polynesia, 2013-15: a retrospective study. Lancet 387, 21 25-2132 (2016).

11. Oliveira Melo, A. S. et al. Zika virus intrauterine infection causes fetal brain abnormality and microcephaly: tip of the iceberg? Ultrasound Obstet. Gynecol. 47, 6-7 (2016).

12. Ventura, C. V., Maia, M., Bravo-Filho, V., Gois, A. L. $\&$ Belfort, R. Zika virus in Brazil and macular atrophy in a child with microcephaly. Lancet 387, 228 (2016)

13. Rasmussen, S. A., Jamieson, D. J., Honein, M. A. ¿ Petersen, L. R. Zika virus and birth defects reviewing the evidence for causality. N. Engl. J. Med. 374, 1981-1987 (2016).

14. Sarno, M. et al. Zika virus infection and stillbirths: a case of hydrops fetalis, hydranencephaly and fetal demise. PLoS Negl. Trop. Dis. 10, e0004517 (2016).

15. Johansson, M. A., Mier-y-Teran-Romero, L., Reefhuis, J., Gilboa, S. M. \& Hills, S. L. Zika and the risk of microcephaly. N. Engl. J. Med. 375, 1-4 (2016).

16. Franca, G. V. et al. Congenital Zika virus syndrome in Brazil: a case series of the first 1501 livebirths with complete investigation. Lancet http://dx.doi. org/10.1016/S0140-6736(16)30902-3 (2016).
17. Corona-Rivera, J. R. et al. Report and review of the fetal brain disruption sequence. Eur. J. Pediatr. 160, 664-667 (2001).

18. Petersen, E. E. et al. Update: interim guidance for health care providers caring for women of reproductive age with possible Zika virus exposure - United States, 2016. MMWR. Morb. Mortal. Wkly Rep. 65, 315-322 (2016).

19. Brasil, P. et al. Zika virus infection in pregnant women in Rio de Janeiro - preliminary report. N. Engl. J. Med. http://dx.doi.org/10.1056/NEJMoa1602412 (2016).

20. Ventura, C. V. et al. Ophthalmological findings in infants with microcephaly and presumable intra-uterus Zika virus infection. Arq. Bras. Oftalmol. 79, 1-3 (2016)

21. Chan, J. F., Choi, G. K., Yip, C. C., Cheng, V. C. \& Yuen, K. Y. Zika fever and congenital Zika syndrome: An unexpected emerging arboviral disease. J. Infect. 72, 507-524 (2016).

22. Hustin, J. \& Schaaps, J. P. Echographic [corrected] and anatomic studies of the maternotrophoblastic border during the first trimester of pregnancy. $A m$. $J$. Obstet. Gynecol. 157, 162-168 (1987).

23. Foidart, J. M., Hustin, J., Dubois, M. \& Schaaps, J. P. The human placenta becomes haemochorial at the 13th week of pregnancy. Int. J. Dev. Biol. 36, 451-453 (1992)

24. Burton, G. J., Jauniaux, E. \& Watson, A. L. Maternal arterial connections to the placental intervillous space during the first trimester of human pregnancy: the Boyd collection revisited. Am. J. Obstet. Gynecol. 181 718-724 (1999).

25. Nahmias, A. J. et al. Perinatal risk associated with maternal genital herpes simplex virus infection. Am. J. Obstet. Gynecol. 110, 825-837 (1971).

26. Plotkin, S. A. Rubella eradication. Vaccine 19, 3311-3319 (2001)

27. Schantz-Dunn, J. \& Nour, N. M. Malaria and pregnancy: a global health perspective. Rev. Obstet. Gynecol. 2, 186-192 (2009)

28. Martines, R. B. et al. Pathology of congenital Zika syndrome in Brazil: a case series. Lancet http://dx.doi. org/10.1016/S0140-6736(16)30883-2 (2016).

29. Pacheco, O. et al. Zika virus disease in Colombia preliminary report. N. Engl. J. Med. http://dx.doi. org/10.1056/NEJMoa1604037 (2016).

30. Ventura, C. V., Maia, M., Dias, N., Ventura, L. O. $\&$ Belfort, R. Zika: neurological and ocular findings in infant without microcephaly. Lancet 387, 2502 (2016).

31. US National Institutes of Health. NIH launches large study of pregnant women in areas affected by Zika virus. NIH https://www.nih.gov/news-events/newsreleases/nih-launches-large-study-pregnant-womenareas-affected-zika-virus (2016)

32. Robbins, J. R., Skrzypczynska, K. M., Zeldovich, V. B., Kapidzic, M. \& Bakardjiev, A. I. Placental syncytiotrophoblast constitutes a major barrier to vertical transmission of Listeria monocytogenes. PLoS Pathog. 6, e 1000732 (2010).

33. Robbins, J. R., Zeldovich, V. B., Poukchanski, A Boothroyd, J. C. \& Bakardjiev, A. I. Tissue barriers of the human placenta to infection with Toxoplasma gondii. Infect. Immun. 80, 418-428 (2012).

34. Bayer, A. et al. Human trophoblasts confer resistance to viruses implicated in perinatal infection. Am. J. Obstet. Gynecol. 212, 71 (2015).

35. Delorme-Axford, E. et al. Human placental trophoblasts confer viral resistance to recipient cells. Proc. Natl Acad. Sci. USA 110, 12048-12053 (2013).

36. Bayer, A. et al. Type III interferons produced by human placental trophoblasts confer protection against Zika virus infection. Cell Host Microbe 19, 705-712 (2016).

37. Zeldovich, V. B. et al. Placental syncytium forms a biophysical barrier against pathogen invasion. PLoS Pathog. 9, e1003821 (2013).

38. Tabata, T. et al. Zika virus targets different primary human placental cells, suggesting two routes for vertical transmission. Cell Host Microbe http://dx.doi. org/10.1016/i.chom.2016.07.002 (2016)

39. Driggers, R. W. et al. Zika virus infection with prolonged maternal viremia and fetal brain abnormalities. N. Engl. J. Med. 374, 2142-2151 (2016)

40. Miner, J. J. et al. Zika virus infection during pregnancy in mice causes placental damage and fetal demise. Cell 165, 1081-1091 (2016)

41. Maltepe, E., Bakardjiev, A. I. \& Fisher, S. J. The placenta: transcriptional, epigenetic, and physiological integration during development. J. Clin. Invest. 120 1016-1025 (2010)
42. Kim, K. \& Shresta, S. Neuroteratogenic viruses and lessons for Zika virus models. Trends Microbiol. 24 , 622-636 (2016)

43. Quicke, K. M. et al. Zika virus infects human placental macrophages. Cell Host Microbe 20 83-90 (2016).

44. Adams, K. M. \& Nelson, J. L. Microchimerism: an investigative frontier in autoimmunity and transplantation. JAMA 291, 1127-1131 (2004)

45. Maidji, E., McDonagh, S., Genbacev, O., Tabata, T. \& Pereira, L. Maternal antibodies enhance or prevent cytomegalovirus infection in the placenta by neonatal Fc receptor-mediated transcytosis. Am. J. Pathol. 168 1210-1226 (2006)

46. Guzman, M. G., Alvarez, M. \& Halstead, S. B. Secondary infection as a risk factor for dengue hemorrhagic fever/dengue shock syndrome: an historical perspective and role of antibodydependent enhancement of infection. Arch. Virol. 158, 1445-1459 (2013)

47. de Alwis, R. et al. Dengue viruses are enhanced by distinct populations of serotype cross-reactive antibodies in human immune sera. PLoS Pathog. 10, e 1004386 (2014)

48. Castanha, P. M. et al. Placental transfer of dengue virus (DENV)-specific antibodies and kinetics of DENV infection-enhancing activity in Brazilian infants. J. Infect. Dis. 214, 265-272 (2016).

49. Lanciotti, R. S. et al. Genetic and serologic properties of Zika virus associated with an epidemic, Yap State, Micronesia, 2007. Emerg. Infect. Dis. 14, 1232-1239 (2008).

50. Dejnirattisai, W. et al. Dengue virus sero-crossreactivity drives antibody-dependent enhancement of infection with Zika virus. Nat. Immunol. http://dx.doi. org/10.1038/ni.3515 (2016)

51. Priyamvada, L. et al. Human antibody responses after Dengue virus infection are highly cross-reactive to Zik virus. Proc. Natl Acad. Sci. USA 113, 7852-7857 (2016).

52. Swanstrom, J. A. et al. Dengue virus envelope dimer epitope monoclonal antibodies isolated from dengue patients are protective against Zika virus. $\mathrm{mBio} 7$ e01123-16 (2016)

53. Stettler, K. et al. Specificity, cross-reactivity and function of antibodies elicited by Zika virus infection. Science http://dx.doi.org/10.1126/science.aaf8505 (2016).

54. Dancis, J., Lind, J., Oratz, M., Smolens, J. \& Vara, P. Placental transfer of proteins in human gestation. Am. J. Obstet. Gynecol. 82, 167-171 (1961).

55. Bright, N. A. \& Ockleford, C. D. Cytotrophoblast cells: a barrier to maternofetal transmission of passive immunity. J. Histochem. Cytochem. 43, 933-944 (1995).

56. Gusdon, J. P. Fetal and maternal immunoglobulin levels during pregnancy. Am. J. Obstet. Gynecol. 103 895-900 (1969).

57. Garty, B. Z., Ludomirsky, A., Danon, Y. L., Peter, J. B. \& Douglas, S. D. Placental transfer of immunoglobulin G subclasses. Clin. Diagn. Lab Immunol. 1, 667-669 (1994).

58. Malek, A., Sager, R., Kuhn, P., Nicolaides, K. H. $\Sigma$ Schneider, H. Evolution of maternofetal transport of immunoglobulins during human pregnancy. Am. J. Reprod. Immunol. 36, 248-255 (1996)

59. Dudley, D. M. et al. A rhesus macaque model of Asianlineage Zika virus infection. Nat. Commun. 7, 12204 (2016).

60. Prisant, N. et al. Zika virus in the female genital tract. Lancet Infect. Dis. http://dx.doi.org/10.1016/S14733099(16)30193-1 (2016)

61. Mlakar, J. et al. Zika virus associated with microcephaly. N. Engl. J. Med. 374, 951-958 (2016).

62. Qian, X. et al. Brain-region-specific organoids using mini-bioreactors for modeling ZIKV exposure. Cell 165, 1238-1254 (2016)

63. Hanners, N. W. et al. Western Zika virus in human fetal neural progenitors persists long term with partial cytopathic and limited immunogenic effects. Cell Rep. 15, 2315-2322 (2016)

64. Tang, H. et al. Zika virus infects human cortical neural progenitors and attenuates their growth Cell Stem Cell 18, 587-590 (2016).

65. Garcez, P. P. et al. Zika virus impairs growth in human neurospheres and brain organoids. Science 352 , 816-818 (2016)

66. Dang, J. et al. Zika virus depletes neural progenitors in human cerebral organoids through activation of the innate immune receptor TLR3. Cell Stem Cell http./ dx.doi.org/10.1016/j.stem.2016.04.014 (2016). 
67. Cugola, F. R. et al. The Brazilian Zika virus strain causes birth defects in experimental models. Nature 534, 267-271 (2016).

68. Lazear, H. M. et al. A mouse model of Zika virus pathogenesis. Cell Host Microbe 19, 720-730 (2016).

69. Rossi, S. L. et al. Characterization of a novel murine model to study Zika virus. Am. J. Trop. Med. Hyg. 94 1362-1369 (2016).

70. Aliota, M. T. et al. Characterization of lethal Zika virus infection in AG129 mice. PLoS Negl. Trop. Dis. 10 e0004682 (2016).

71. Dowall, S. D. et al. A susceptible mouse model for Zika virus infection. PLoS Negl. Trop. Dis. 10, e0004658 (2016).

72. Wu, K. Y. et al. Vertical transmission of Zika virus targeting the radial glial cells affects cortex development of offspring mice. Cell Res. 26, 645-654 (2016).

73. Schmidt, A., Morales-Prieto, D. M., Pastuschek, J., Frohlich, K. \& Markert, U. R. Only humans have human placentas: molecular differences between mice and humans. J. Reprod. Immunol. 108, 65-71 (2015).

74. Wack, A., Terczynska-Dyla, E. \& Hartmann, R. Guarding the frontiers: the biology of type III interferons. Nat. Immunol. 16, 802-809 (2015).

75. Li, C. et al. Zika virus disrupts neural progenitor development and leads to microcephaly in mice. Cell Stem Cell 19, 120-126 (2016).

76. Dick, G. W. Zika virus. II. Pathogenicity and physical properties. Trans. R. Soc. Trop. Med. Hyg. 46, 521-534 (1952)

77. Paixao, E. S., Teixeira, M. G., Costa, M. D \& Rodrigues, L. C. Dengue during pregnancy and adverse fetal outcomes: a systematic review and meta-analysis. Lancet Infect. Dis. 16, 857-865 (2016).

78. Shepard, D. S., Undurraga, E. A., Halasa, Y. A. $\&$ Stanaway, J. D. The global economic burden of dengue: a systematic analysis. Lancet Infect. Dis. 16, 935-941 (2016).

79. Bhatt, S. et al. The global distribution and burden of dengue. Nature 496, 504-507 (2013).

80. Kinney, J. S. \& Kumar, M. L. Should we expand the TORCH complex? A description of clinical and diagnostic aspects of selected old and new agents. Clin. Perinatol. 15, 727-744 (1988).

81. Musso, D. et al. Potential sexual transmission of Zika virus. Emerg. Infect. Dis. 21, 359-361 (2015).
82. Hills, S. L et al. Transmission of Zika virus through sexual contact with travelers to areas of ongoing transmission - continental United States, 2016. MMWR. Morb. Mortal. Wkly Rep. 65, 215-216 (2016).

83. Deckard, D. T. et al. Male-to-male sexual transmission of Zika virus - Texas, January 2016. MMWR. Morb. Mortal. Wkly Rep. 65, 372-374 (2016).

84. D'Ortenzio, E. et al. Evidence of sexual transmission of Zika virus. N. Engl. J. Med. 374, 2195-2198 (2016).

85. Foy, B. D. et al. Probable non-vector-borne transmission of Zika virus, Colorado, USA. Emerg. Infect. Dis. 17 880-882 (2011).

86. Davidson, A., Slavinski, S., Komoto, K., Rakeman, J. \& Weiss, D. Suspected female-to-male sexual transmission of Zika virus - New York City, 2016. MMWR. Morb. Mortal. Wkly Rep. 65, 716-717 (2016).

87. Mansuy, J. M. et al. Zika virus: high infectious viral load in semen, a new sexually transmitted pathogen? Lancet Infect. Dis. 16, 405 (2016).

88. Duffy, M. R. et al. Zika virus outbreak on Yap Island, Federated States of Micronesia. N. Engl. J. Med. 360, 2536-2543 (2009).

89. Besnard, M. et al. Congenital cerebral malformations and dysfunction in fetuses and newborns following the 2013 to 2014 Zika virus epidemic in French Polynesia. Euro Surveill. http://dx.doi.org/10.2807/1560-7917. ES.2016.21.13.30181 (2016).

90. Liotta, G. et al. Elimination of mother-to-child transmission of HIV infection: the drug resource enhancement against AIDS and malnutrition model. Int. J. Environ. Res. Public Health 12, 13224-13239 (2015).

91. Stevens, J. \& Lyall, H. Mother to child transmission of HIV: what works and how much is enough? J. Infect. 69 (Suppl. 1), S56-S62 (2014).

92. Perkins, T. A., Siraj, A. S., Ruktanonchai, C. W. Kraemer, M. U. G. \& Tatem, A. J. Model-based projections of Zika virus infections in childbearing women in the Americas. Nat. Microbiol. 1, 16126 (2016).

93. Lambert, N., Strebel, P., Orenstein, W., Icenogle, J. \& Poland, G. A. Rubella. Lancet 385, 2297-2307 (2015)

94. Manicklal, S., Emery, V. C., Lazzarotto, T., Boppana, S. B. \& Gupta, R. K. The "silent" global burden of congenital cytomegalovirus. Clin. Microbiol. Rev. 26, 86-102 (2013).

95. Torgerson, P. R. \& Mastroiacovo, P. The global burden of congenital toxoplasmosis: a systematic review. Bull. World Health Organ. 91, 501-508 (2013).
96. Rossant, J. \& Cross, J. C. Placental development: lessons from mouse mutants. Nat. Rev. Genet. 2, 538-548 (2001)

97. Schleiss, M. R. Developing a vaccine against congenital cytomegalovirus (CMV) infection: what have we learned from animal models? where should we go next? Future Virol. 8, 1161-1182 (2013).

98. Carter, A. M. Animal models of human placentation a review. Placenta 28 (Suppl. A), S41-S47 (2007).

99. World Health Organization. Zika situation report. WHO http://www,who.int/emergencies/zika-virus/ situation-report/14-july-2016/en/ (2016).

100. Perez, S. et al. Confirmed case of Zika virus congenita infection, Spain, March 2016. Euro Surveill. http://dx. doi.org/10.2807/1560-7917.ES.2016.21.24.30261 (2016).

101. Culjat, M. et al. Clinical and imaging findings in an infant with Zika embryopathy. Clin. Infect. Dis. http:// dx.doi.org/10.1093/cid/ciw324 (2016)

102. Aiken, A. R. et al. Requests for abortion in Latin America related to concern about Zika virus exposure N. Engl. J. Med. 375, 396-398 (2016).

103. Oster, A. M. et al. Update: interim guidance for prevention of sexual transmission of Zika virus United States, 2016. MMWR. Morb. Mortal. Wkly Rep. 65, 323-325 (2016)

104. Guttmacher Institute. Facts on abortion in Latin America and the Caribbean. Guttmacher Institute https://www.guttmacher.org/fact-sheet/facts-abortionlatin-america-and-caribbean (2015)

105. McConkey, C. A. et al. A three-dimensional culture system recapitulates placental syncytiotrophoblast development and microbial resistance. Sci. Adv. 2, e1501462 (2016).

\section{Acknowledgements}

The authors apologize to those whose work was not covered in detail owing to space restrictions and for inaccuracies or omissions resulting from the remarkably rapid pace of discovery in the Zika virus field. The authors thank Y. Sadovsky for helpful discussions in relation to this manuscript. C.B.C is supported by the US National Institutes of Health (NIH; grants R01-Al081759 and NIH R01-HD075665), and the Burroughs Wellcome Investigators in the Pathogenesis of Infectious Disease Award. H.M.L is supported by start-up funds from the University of North Carolina Department of Microbiology and Immunology and the Lineberger Comprehensive Cancer Center.

Competing interests statement

The authors declare no competing interests. 